\title{
Multifunctional polymer-containing coatings on magnesium alloys
}

\author{
Dmitry $V$. Mashtalyar ${ }^{1,2, *}$, Konstantine $V$. Nadaraia ${ }^{1,2}$, and Sergey $V$. Gnedenkov ${ }^{1}$ \\ ${ }^{1}$ Institute of Chemistry Far Eastern Branch of Russian Academy of Sciences, 690022 Vladivostok, 159 100-letiya Vladivostoka Pr., \\ Russia \\ ${ }^{2}$ Far Eastern Federal University, 690950 Vladivostok, 8 Sukhanova St., Russia
}

\begin{abstract}
The results of surface modification of magnesium alloys by plasma electrolytic oxidation (PEO) and post-treatment in suspension of tetrafluoroethylene (TFE) telomeric solution or superdispersed polytetrafluoroethylene (SPTFE) dispersion have been presented. Electrochemical, tribological properties and wetting ability of obtained protective composite coatings were investigated. The fabricated coatings decrease both the corrosion current density $\left(1.7 \times 10^{-9}-5.4 \times 10^{-11} \mathrm{~A} / \mathrm{cm}^{2}\right)$ and wear $\left(9.8 \times 10^{-6}-7.6 \times 10^{-7}\right.$ $\left.\mathrm{mm}^{3} /(\mathrm{N} \mathrm{m})\right)$ by orders of magnitude as compared to bare magnesium alloy $\left(5.3 \times 10^{-5} \mathrm{~A} / \mathrm{cm}^{2}\right.$ and $3.8 \times 10^{-3}$ $\left.\mathrm{mm}^{3} /(\mathrm{N} \mathrm{m})\right)$ and base PEO-coating $\left(2.4 \times 10^{-7} \mathrm{~A} / \mathrm{cm}^{2}\right.$ and $\left.1.7 \times 10^{-3} \mathrm{~mm}^{3} /(\mathrm{N} \mathrm{m})\right)$. Moreover, coatings obtained by means of PEO and polymer dispersion dipping possess hydrophobic and superhydrophobic properties. Such multifunctional coatings have high corrosion resistance and good friction coefficient under dry wear conditions that extends the application field of magnesium alloys.
\end{abstract}

\section{Introduction}

Plasma electrolytic oxidation (PEO) is an advanced surface modification process, which leads to the formation of similar to ceramic and well-adhered coatings on the valve metals such as aluminium, magnesium, titanium, and a wide range of their alloys [1-4]. There are many advantages of PEO-coatings, such as a high hardness, corrosion and wear resistances and biocompatibility. PEO-layers provide better protection than conventional anodic oxide films, which makes PEO suitable for many industrial applications. In spite of many advantages, the PEO-coatings can have structural defects such as cracks and pores, which could dramatically reduce the corrosion resistance of even relatively thick coatings. There are several attempts to reduce or seal such porosity. The post-treatments are often applied in order to seal the pores and improve the protection effect in practical industrial applications where the PEO-layer is used as a base for composite coating formation [5-7].

In recent years, there have been numerous reports in the literature about PEO-coatings on $\mathrm{Mg}$ alloy with post-treatments. The post-treatments of PEO-based coatings can usually exhibit significantly better corrosion protection ability than the single PEO-coatings, suggesting better application prospects.

PEO allows forming on the magnesium alloy protective layers, which have a convolute, rough surface. Such structure can be served as a basis for application at the PEO-surface polymeric materials in order to provide additional protection for this product $[8,9]$. In this regard, polymers due to their chemical stability represent a particular interest. This paper describes the results of investigation of protective properties of multifunctional composite coatings (CC) on magnesium alloys with various polymer.

\section{Methods}

Rectangular plates of a size of $15 \mathrm{~mm} \times 20 \mathrm{~mm} \times 2 \mathrm{~mm}$ made of the magnesium alloy MA8 (1.5 wt. \% Mn; 0.15 wt. \% Ce; $\mathrm{Mg}$ - balance) were used as samples. Prior to oxidation, for the surface standardization, the samples underwent mechanical treatment by grinding paper of different grain sizes $(600,800$, and 1200$)$, washed with distilled water, and degreased with ethanol. Silicatefluoride solution was used as electrolyte for PEO [3, 68]. The process of coating formation was realized using the tyristor rectifier as a power supply. Coatings were formed in bipolar PEO-mode. In the first step the anodic current density was specified of $0.61 \mathrm{~A} \cdot \mathrm{cm}^{-2}$ (voltage increases up to $270 \mathrm{~V}$ ), cathodic component was kept potentiostatically at $-40 \mathrm{~V}$. In the second step the voltage was gradually changed down to $200 \mathrm{~V}$ in the anodic part and up to $-10 \mathrm{~V}$ in the cathodic one. The duration of the first and second phases of the PEO was $200 \mathrm{~s}$ and $600 \mathrm{~s}$, respectively.

Two different solutions for the composite coating formation were used. TFE telomeric solution in acetone and aqueous suspension of SPTFE (trademark Forum ${ }^{\circledR}$ ) were used as a polymer-containing component for formation of composite coatings. The polymer was deposited by dipping of the sample with PEO-coating in

\footnotetext{
* Corresponding author: madiva@inbox.ru
} 
one of two used solutions. Time of the immersion in suspensions was not more than $10-15 \mathrm{~s}$. After application, the samples were air dried for 30 minutes at $25^{\circ} \mathrm{C}$. Next step was heat treatment at $250{ }^{\circ} \mathrm{C}$ for coatings, which were obtained by application of telomeric solution (the number of applications was 3 ) (hereinafter $\mathrm{CC} \# 1$ ) and at $315^{\circ} \mathrm{C}$ for coatings, which were obtained by application of SPTFE (the number of applications was 4) (hereinafter $\mathrm{CC} \# 2$ ).

Electrochemical properties of coatings were investigated using electrochemical system VersaSTAT MC (Princeton Applied Research, USA). Measurements were carried out in a three-electrode cell at room temperature in a $3 \% \mathrm{NaCl}$ solution. Platinum mesh was used as a counter electrode. Calomel electrode was used as a reference electrode. The exposed area of the sample was equal to $1 \mathrm{~cm}^{2}$. Potentiodynamic measurements were carried out at sweep rate of $5 \mathrm{mV} \mathrm{s}^{-1}$ from $E_{\mathrm{c}}-0.15$ $\mathrm{V}$ up to $E_{\mathrm{c}}+0.5 \mathrm{~V}$. During the impedance measurements, sinusoidal signal with amplitude of 10 $\mathrm{mV}$ was used. A spectrum was recorded at the open circuit potential $\left(E_{\mathrm{c}}\right)$ in the frequency range from 0.01 $\mathrm{Hz}$ to $0.1 \mathrm{MHz}$.

The wettability of the obtained composite coating was evaluated by sessile drop method on Drop Shape Analyser DSA100 (KRÜSS, Germany). Solution of $3 \%$ $\mathrm{NaCl}$ in distilled water was used for contact angle measurements.

Tribological tests were performed using a Tribometer (CSM Instruments, Switzerland) in accordance with the 'ball-on-plate' scheme. A ball of a diameter of $10 \mathrm{~mm}$ made of alumina was used as a counterbody. All the studies were carried out under dry friction and ambient conditions $\left(25{ }^{\circ} \mathrm{C}\right.$ and relative humidity of $\left.50 \%\right)$. A normal load was equal to $10 \mathrm{~N}$. The linear rotation speed was equal to $50 \mathrm{~mm} / \mathrm{s}$, the track diameter - to $10 \mathrm{~mm}$.

The surface morphology of the obtained composite coating was studied with use a Scanning Electron Microscope (SEM) EVO 40 (Carl Zeiss, Germany).

\section{Results and discussions}

Data analysis of SEM-images identified the influence of applying polymer and subsequent heat treatment on the surface morphology of the formed layers (Fig. 1). At surface treatment of the base PEO-coating (Fig. 1a) with the telomeric solution the number of pores and defects on the surface decrease and the surface becomes more uniform (Fig. 1b). In the case of applying SPTFE (Fig. 1c) a uniform film was formed with no visible defects.

The potentiodynamic polarization curves and calculated corrosion parameters are presented in Fig. 2 and Table, respectively. These results enable one to conclude that application of polymer at the surface of the base PEO-coating has a positive effect on the protective properties of the both formed composite layers.

In the case of applying at the surface treatment the TFE telomeric solution the corrosion current density $\left(I_{c}\right)$ reduces, and the polarization resistance $\left(R_{p}\right)$ increases by 4 orders of magnitude as compared to the uncoated material and by 2 orders of magnitude as compared to the PEO-coating (see Table).
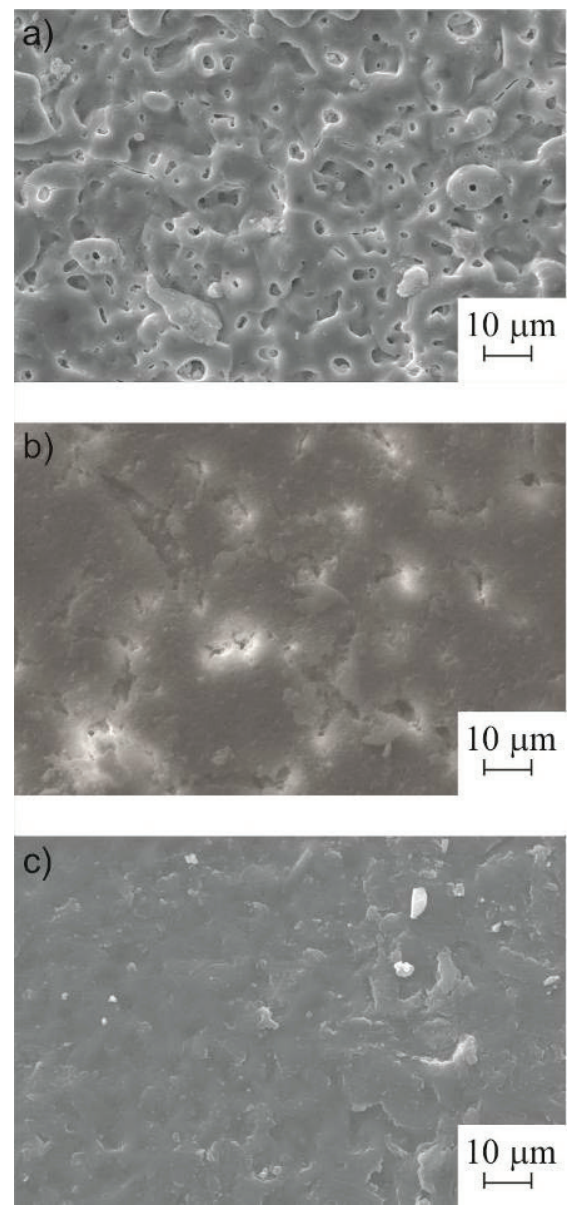

Fig. 1. SEM-image of PEO-coating (a) and composite coatings obtained using the TFE telomeric solution (b) and suspension of SPTFE (c)

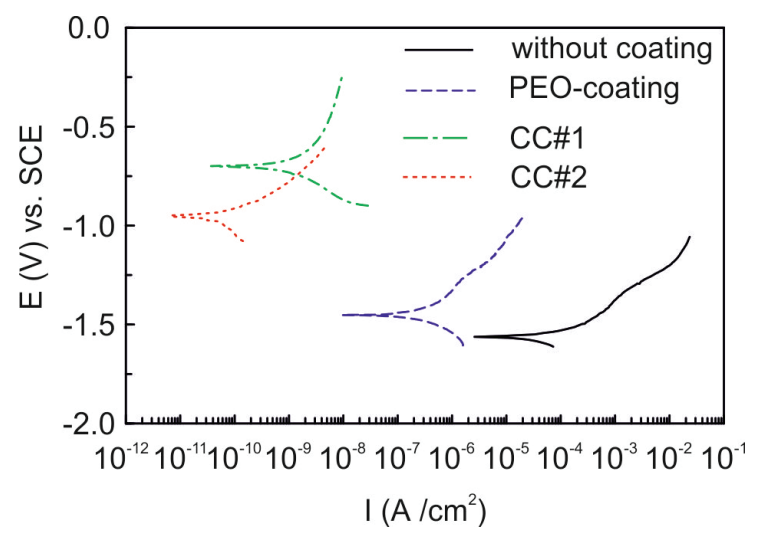

Fig. 2. Polarisation curves obtained in $3 \% \mathrm{NaCl}$ solution for the studied samples without coating (solid line), with PEOcoating (dashed line), with composite coating obtained with triple application of TFE solution (CC\#1, dashed-dotted line) and with composite coating obtained with fourfold application of SPTFE (CC\#2, dotted line).

Formed coatings, which were obtained using SPTFE suspension, have even higher protective properties. For such composite coating $I_{\mathrm{c}}$ reduces and $R_{\mathrm{p}}$ increases by 6 and 4 orders of magnitude as compared to the uncoated alloy and PEO-coating, respectively (see Table). 
Moreover, for both types of composite layers the value of $E_{\mathrm{c}}$ increases at least $0.5 \mathrm{~V}$ as compared to one for the PEO-coating. The conclusion can be done that $\mathrm{CC} \# 1$ has lower values of $R_{\mathrm{p}}$, and a higher $I_{\mathrm{c}}$ as compared to CC\#2 (see Table). Apparently, this is due to the greater uniformity of the film obtained using a suspension SPTFE (Fig. 1c) as compared to the film obtained using telomeric solution (Fig. 1b).

Analysis of electrochemical impedance spectroscopy data revealed a significant difference in the morphology of the composite coatings depending on type of polymer. Observed transformation of the peak position and amplitude of the phase angle (Fig. 3b) indicats about the changes in the surface state: porosity, homogeneous of composite layers.
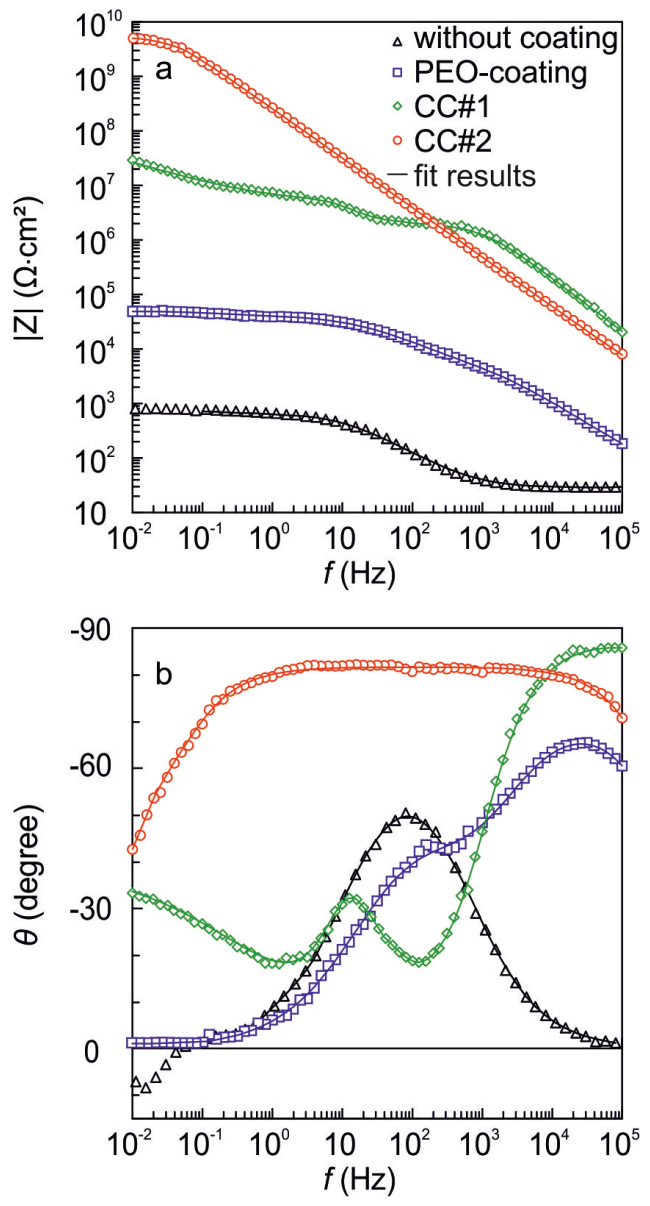

Fig. 3. Bode plots (dependence of the impedance modulus $|Z|_{f}$ $\rightarrow 0 \mathrm{~Hz}$ (a) and the phase angle $\theta$ (b) on the frequency $f$ ) for the studied samples without coating (square symbols), with PEOcoating (triangle symbols), with composite coating obtained with triple application of TFE solution (CC\#1, diamond symbols) and with composite coating obtained with fourfold application of SPTFE (CC\#2, circle symbols). Impedance spectra contain experimental data (scatter plot marked by symbols) and theoretical fitting curves (lines), which simulate the experimental results by means of equivalent electrical circuits.

In order to describe the charge transfer mechanism at the coating/electrolyte interface, three kinds of equivalent electrical circuits (EEC) were used. For the uncoated sample the one-R-CPE-circuit was applied (Fig. 4a). For samples with coatings two kinds of EEC were used: two $R-C P E$-chain circuit (base PEO-coating) (Fig. 4b) and three $R-C P E$-chain circuit (composite coatings) (Fig. 4c).

The using three- $R-C P E$-chain circuit associated with the appearance of the third time constant on the spectra (Fig. 3b). This is a consequence of the sealing air by polymeric plug in the pores of PEO-layer. It should be noted that the dependence of the phase angle $\theta$ on the frequency $f$ for $\mathrm{CCH} 2$ (Fig. 3b) is capacitive, which suggests a high uniformity of the obtained coating. According to fitting of experimental impedance spectra by a presented EES it was established that TFE- and SPTFE-treatment of the PEO-coating results in the increase of the thickness of the inner porousless sublayer and the geometric thickness of the coating as well.

The results of experiments enable one to conclude that multistep treatment of the PEO coated sample with telomeric solution increases the impedance modulus $|\mathrm{Z}|_{f \rightarrow 0 \mathrm{~Hz}}$ up to $10^{7} \Omega \cdot \mathrm{cm}^{2}$, and in the case of the treating with suspension of SPTFE up to $10^{9} \Omega \cdot \mathrm{cm}^{2}$, that is almost by 3 and 5 orders of magnitude higher than one for the PEO-coating (Fig. 3a, Table). Apparently, all abovementioned reasons of improving the characteristics of composite coating associated with the sealing the pores and defects, which are in the base PEO-coating.

Results of wettability investigations for samples revealed the high hydrophobic properties of CCs (see Table). The highest values of the contact angle $\left(171^{\circ}\right)$ were obtained for the CC\#1. Contact angle values of greater than $150^{\circ}$ indicate that this sample possesses the superhydrophobic properties. In accordance with the results of previous studies a superhydrophobicity is caused by multimodal roughness of surface. Apparently, in the case of using of the TFE telomeric solution such structure is formed as a result of the partial destruction of the polymer compounds and subsequent aggregation during heat treatment.

As a result of data analysis about changing the friction coefficient during test (Fig. 5) there are at least 2 stages of the wear for composite polymer containing coatings. The first stage is an abrasion of polymer film. In this stage the friction coefficient $\mu$ varies from 0.10 to 0.15 (Fig. 5) in dependence on the forming method of the composite layer. The second stage (abrasion of the PEO-layer) is characterised by a sharp increase of the friction coefficient (Fig. 5).

At full abrasion of the coating in the contact zone, the friction coefficient attains of 0.55 and more. Analysis of tribological data revealed a positive impact of incorporation of polymer particles into the PEO-coating to improve the wear resistance of composite layers (Tab.). Thus, the applying of TFE telomeric solution reduces wear of the $\mathrm{CC \# 1}$ by 3 orders of magnitude as compared to the base PEO-coating. For composite coating, obtained using SPTFE suspension the wear was decreased by 4 orders of magnitude in comparison with the base PEO-layer. Such increasing of the wear resistance is associated with the formation of the polymer-containing layer in the outer part of the PEOcoating, which has a low friction coefficient and acts as a dry lubricant. 
a)

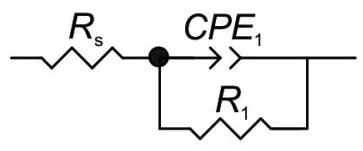

b)

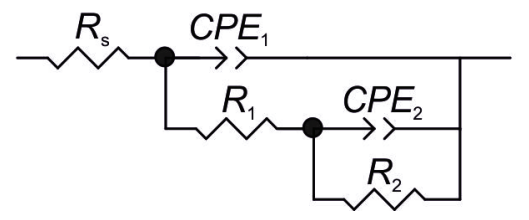

c)

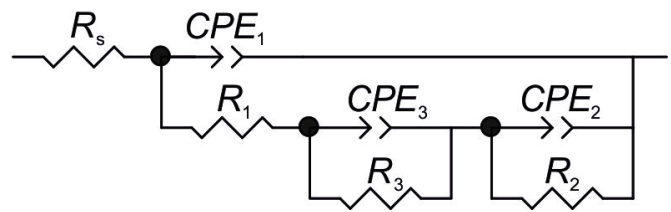

Fig. 4. The equivalent electrical circuits used for the fit of the experimental impedance spectra: one- $R-C P E$-chain EEC (a); two- $R-$ $C P E$-chain EEC (b); three- $R-C P E$-chain EEC (c).

Table 1. Corrosion, tribological properties, and wettability of investigated samples

\begin{tabular}{|c|c|c|c|c|c|c|}
\hline Type of coating & $\begin{array}{c}\boldsymbol{R}_{\mathbf{p}} \\
\left(\boldsymbol{\Omega} \cdot \mathbf{c m}^{\mathbf{2}}\right)\end{array}$ & $\begin{array}{c}\boldsymbol{E}_{\mathbf{C}} \\
(\mathbf{V} \text { vs. SCE) }\end{array}$ & $\begin{array}{c}\boldsymbol{I}_{\mathbf{C}} \\
\left(\mathbf{A} / \mathbf{c m}^{\mathbf{2}}\right)\end{array}$ & $\begin{array}{c}|\mathbf{Z}|_{\mathbf{f} \rightarrow \mathbf{0 ~ H z}} \\
\left(\boldsymbol{\Omega} \mathbf{~ c m}^{\mathbf{2}}\right)\end{array}$ & $\begin{array}{c}\mathbf{C A} \\
\left({ }^{\circ}\right)\end{array}$ & $\begin{array}{c}\mathbf{W e a r} \mathbf{r a t e} \\
\left(\mathbf{m m}^{\mathbf{3}} /(\mathbf{N} \cdot \mathbf{m})\right)\end{array}$ \\
\hline Without coating & $(0.5 \pm 0.1) \times 10^{3}$ & $-1.61 \pm 0.07$ & $(5.3 \pm 0.2) \times 10^{-5}$ & $(0.8 \pm 0.03) \times 10^{3}$ & $38 \pm 1$ & $(3.8 \pm 0.2) \times 10^{-3}$ \\
\hline Base PEO & $(2,8 \pm 0.3) \times 10^{5}$ & $-1.48 \pm 0.06$ & $(2.4 \pm 0.07) \times 10^{-7}$ & $(4.3 \pm 0.1) \times 10^{4}$ & $49 \pm 2$ & $(1.7 \pm 0.09) \times 10^{-3}$ \\
\hline CC\#1 & $(3.2 \pm 0.5) \times 10^{7}$ & $-0.70 \pm 0.02$ & $(1.7 \pm 0.05) \times 10^{-9}$ & $(2.9 \pm 0.09) \times 10^{7}$ & $171 \pm 2$ & $(9.8 \pm 0.5) \times 10^{-6}$ \\
\hline CC\#2 & $(4.9 \pm 0.4) \times 10^{8}$ & $-0.95 \pm 0.05$ & $(5.4 \pm 0.2) \times 10^{-11}$ & $(1.9 \pm 0.06) \times 10^{9}$ & $140 \pm 2$ & $(7.6 \pm 0.4) \times 10^{-7}$ \\
\hline
\end{tabular}

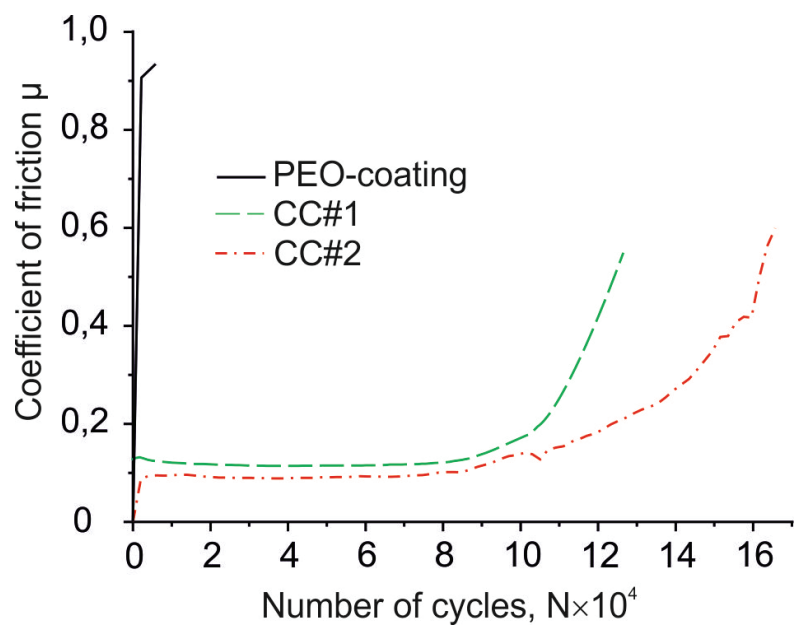

Fig. 5. Dependence of the friction coefficient on the number of cycles for samples made of magnesium alloy MA8 with PEOcoating (solid line), with composite coating obtained with triple application of TFE solution (CC\#1, dotted line) and with composite coating obtained with fourfold application of SPTFE (CC\#2, dashed-dotted line).

\section{Conclusion}

New way of the protective composite layers formation using PEO method and subsequent treatment of PEOcoating with fluoropolymer materials was developed. The obtained polymer-containing coatings decrease significantly the corrosion current density and the wear, as compared to unprotected alloy. Values of the contact angle attain up to $171^{\circ}$.

According to electrochemical impedance spectroscopy data and calculated parameters of the elements of equivalent electrical circuits, the SPTFEand TFE-treatment of the PEO-coating results in the increase of the thickness of the inner porousles sublayer and the composite coating in whole.

This work was supported by the Russian Science Foundation (№ 14-33-00009).

\section{References}

1. S.L. Sinebryukhov, A.S. Gnedenkov, O.A. Khrisanfova, S.V. Gnedenkov, Surf. Eng. 25 565569 (2009)

2. C.S. Dunleavy, I.O. Golosnoy, J.A. Curran, T.W. Clyne, Surf. Coat. Technol. 203 3410-3419 (2009)

3. J. Liang, P.B. Srinivasan, C. Blawert, W. Dietzel, Corros. Sci. 52 540-547 (2010)

4. H. Duan, K. Du, C. Yan, F. Wang, Electrochim. Acta 51 2898-2908 (2006)

5. Z. Yan, M. Wu, H. Qin, Y. He, X. Yu, L. Chen, Surf. Coat. Technol. 209 331-336 (2017)

6. M. Mohedano, E. Matykina, R. Arrabal, B. Mingo, A. Pardo, Appl. Surf. Sci. 346 57-67 (2015)

7. Z. Wang, L. Wu, Y. Qi, W. Cai, Z. Jiang, Surf. Coat. Technol. 204 3315-3318 (2010)

8. S.V. Gnedenkov, S.L. Sinebryukhov, D.V. Mashtalyar, V.M. Buznik, A.M. Emel'yanenko, L.B. Boinovich, Prot. Met. Phys. Chem. Surf. 47 93101 (2011)

9. S.V. Gnedenkov, S.L. Sinebryukhov, D.V. Mashtalyar, K.V. Nadaraia, A.S. Gnedenkov, V.M. Bouznik, Corros. Sci. 111 175-185 (2016) 